\title{
Influence of proton pump inhibitor therapy on intestinal inflammation assessed by fecal calprotectin in pediatric patients
}

\author{
Su Yeong Kim, MD', Na Mi Lee, MD, PhD ${ }^{1,2}$, Sin Weon Yun, MD, PhD ${ }^{1,2}$, Soo Ahn Chae, MD, PhD ${ }^{1,2}$, In Seok Lim, MD, PhD ${ }^{1,2}$, Eung Sang \\ Choi, MD, PhD ${ }^{3}$, Dae Yong Yi, MD, PhD ${ }^{1,2}$ \\ ${ }^{1}$ Department of Pediatrics, Chung-Ang University Hospital, Seoul, ${ }^{2}$ College of Medicine, Chung-Ang University, Seoul, ${ }^{3}$ Department of Pediatrics, Hanil General \\ Hospital, Seoul, Korea
}

Background: An increase in the numbers of patients with gastrointestinal symptoms has recently been observed.

Purpose: To investigate the effects of proton pump inhibitor (PPI) therapy on intestinal inflammation in children and adolescents as confirmed by clinical manifestations and objectively assessed by fecal calprotectin (FC) level measurement.

Methods: Consecutive children (aged 3-18 years) who presented with gastrointestinal symptoms and were treated with or without PPI for at least 1 month were enrolled. Patients were divided into PPI and non-PPI groups. The PPI group was further subdivided by treatment duration and type of PPI used. Stool samples were collected for FC evaluation at baseline and after treatment and clinical data and FC levels were compared between the groups.

Results: Fifty-one patients (15 boys, 36 girls) were enrolled in the study. The PPI group included 37 patients, while the non-PPI group included 14 patients. Clinical symptoms were not significantly different. FC levels and laboratory results, including C-reactive protein levels, white blood cell count, and absolute neutrophil count, were not statistically different before versus after PPI treatment. After treatment, FC levels decreased to $8.1 \mathrm{mg} / \mathrm{kg}(-575.4$ to $340.3 \mathrm{mg} / \mathrm{kg})$ in the PPI group and increased to $5.6 \mathrm{mg} / \mathrm{kg}(-460.0$ to $186.9 \mathrm{mg} / \mathrm{kg}$ ) in the non-PPI group compared to those before treatment $(P=0.841)$. The number of patients with increased FC levels was not significantly different between the 2 groups ( $48.6 \%$ vs. $64.3 \%, P=0.363$ ), similar to that observed in patients with an FC level $>50 \mathrm{mg} / \mathrm{kg}(24.3 \%$ and $7.1 \%, P=0.250)$. PPI therapy type and duration did not affect the $F C$ levels ( $P=0.811$ and $P=0.502$, respectively).

Conclusion: Although we aimed to confirm the evidence of intestinal inflammation due to PPI use in children and adolescents through clinical symptoms and FC measurement, no significant changes were observed.

Key words: Proton pump inhibitors, Inflammation, Calprotectin

\section{Introduction}

Recently, in South Korea, an increase in the numbers of patients with gastroesophageal reflux (GER), gastroesophageal reflux disease (GERD), or functional gastrointestinal disorders such as chronic epigastric pain, functional nausea, and functional vomiting is being observed due to changes in diet and lifestyle. ${ }^{1,2)}$ The prevalence of gastrointestinal disorders in children and adolescents is increasing due to obesity, changes in dietary habits, and psychological factors. Additionally, the use of proton pump inhibitors (PPIs) is also increasing. ${ }^{3-5)}$ Gastric acid suppressive therapy with PPI is also used in the management of gastric or duodenal ulcers and
Corresponding author: Dae Yong Yi, MD, PhD

Division of Pediatric Gastroenterology and Hepatology, Department of Pediatrics, Chung-Ang University Hospital, 102, Heukseok-ro, Dongjak-gu, Seoul 06973, Korea

Tel: +82-2-6299-1480

Fax: +82-2-6299-2064

E-mail: meltemp2@hanmail.net https://orcid.org/0000-0002-4168-7131

Received: 7 February, 2019

Revised: 26 May, 2019

Accepted: 2 July, 2019
Copyright @ 2019 by The Korean Pediatric Society

This is an open-access article distributed under the terms of the Creative Commons Attribution NonCommercial License (http://creativecommons.org/ licenses/by-nc/4.0/ which permits unrestricted noncommercial use, distribution, and reproduction in any medium, provided the original work is properly cited. 
Helicobacter pylori disease, as well as GER. PPIs are more potent than histamine receptor antagonists with generally good treatment outcomes. At the same time, the possibility of side effects in the gastrointestinal tract, is high. ${ }^{6-9)}$

Gastric acid is an important factor in suppressing bacterial overgrowth in the small intestine. ${ }^{10}$ It is known that gastric acid suppression therapies, such as use of PPI, can induce bacterial overgrowth in the gastrointestinal tract. ${ }^{6-9)}$ Various studies have reported on gastric acid suppression and bacterial overgrowth; with most being limited to the diagnosis of bacterial overgrowth itself through a hydrogen breath test (HBT) or duodenal aspiration, or by the identification of certain strains such as Clostridium difficile and H. pylori. ${ }^{6,9,11)}$ However, even if there is bacterial overgrowth, it is not always clinical significance such as intestinal inflammation. Nevertheless, few studies have investigated the intestinal inflammation. Although studies to confirm the clinical symptoms associated with bacterial overgrowth have been conducted, there persists a shortage of research involving pediatric patients. Furthermore, previous studies have compared subjective symptoms; however, there has been no objective analysis of intestinal inflammation. ${ }^{12)}$

Analysis of fecal calprotectin $(\mathrm{FC})$ is a noninvasive method of diagnosing the activity of inflammatory bowel diseases (IBDs) such as Crohn disease and ulcerative colitis. ${ }^{13,14)}$ In addition, it is a useful method for the detection of inflammatory conditions in various intestinal diseases such as polyps, necrotizing enterocolitis, and bacterial colitis. $^{14-16)}$

In the present study, we investigated the effects of PPI therapy on intestinal inflammation, in children and adolescents as confirmed by clinical manifestations and objectively assessed by measurement of FC levels.

\section{Methods}

We conducted a prospective study on consecutive children (age, 3-18 years) at the Chung-Ang University Hospital between October 2015 and February 2018. The study protocol was approved by the Institutional Review Board (IRB) of the Chung-Ang University Hospital (IRB No. C2015157), and written informed consent was provided. Patients who presented with GER symptoms (such as chronic epigastric pain, nausea, and vomiting) and were treated with or without PPI for at least 1 month, were enrolled. The treatment duration was not randomized, and patients were treated for 4 or 8 weeks based on the clinical symptoms. The subjects who agreed to participate in the study collected stool samples for evaluation of FC (GEMINI, Stratec Biomedical AG, Birkenfeld, Germany) concentration at baseline and after 4 or 8 weeks of treatment with PPI and FC results were measured up to 2,000 mg/ $/ \mathrm{kg}$. Exclusion criteria were as follows: use of PPI in the previous 3 months, use of other drug such as nonsteroidal anti-inflammatory drug, IBD, gastrointestinal involvement of Henoch-Schönlein purpura, and presence of significant intestinal disease that could be associated with inflammatory changes such as bacterial colitis.

Enrolled patients were divided into 2 groups, PPI group treated with PPI for at least 1 month and non-PPI group treated without the drug. Prokinetic agents, such as domperidone, were used to relieve symptoms in both groups. All subjects were instructed to continue dietary and lifestyle modifications. The subjects in the PPI group were treated with lansoprazole $15 \mathrm{mg}$ (body weight $\leq 30 \mathrm{~kg}$ ), or $30 \mathrm{mg}$ (body weight $>30 \mathrm{~kg}$ or age $\geq 12$ years), or esomeprazole $20 \mathrm{mg}$ for 4 weeks or 8 weeks. Therefore, the PPI group was further subdivided according to the treatment duration (4 weeks vs. 8 weeks) and type of PPI used (lansoprazole vs. esomeprazole).

We collected the following clinical data from the medical records of the patients: patient age, sex, clinical symptoms (epigastric pain, nocturnal abdominal pain, abdominal distension, nausea, vomiting, loose stool, nocturnal defecation, bloody stool, and abdominal tenderness), and laboratory findings (C-reactive protein [CRP] level, white blood cell [WBC] count, and absolute neutrophil count [ANC] from blood) before and after PPI treatment. Clinical data and FC level were analyzed after 4 or 8 weeks depending on the duration of PPI treatment in the PPI group, while the parameters were only analyzed after 8 weeks in the non-PPI group.

Statistical analyses were performed using SPSS ver. 18.0 (SPSS Inc., Chicago, IL, USA). Fisher exact test and Mann-Whitney test were used to analyze differences between groups. Data are presented as median (range) or numbers and the level of statistical significance was set at $P<0.05$.

\section{Results}

\section{Clinical characteristics, symptoms, and laboratory findings}

Initially, 62 patients were included in the study. Of these, 11 patients were excluded because stool samples were submitted after treatment with PPI, and 51 patients ( 15 boys and 36 girls) were finally enrolled in the study (Table 1). Thirty-seven subjects were treated with PPI for 4 or 8 weeks (PPI group) and 14 were treated without PPI (non-PPI group). There were no significant differences in the sex, age, CRP level, WBC count, and ANC between the PPI and non-PPI groups before treatment. After treatment, the laboratory findings were not statistically different between the 2 groups.

Table 2 shows the comparison of the number of patients who demonstrated worsened laboratory findings and clinical manifestations after PPI therapy. The proportion of patients who had elevated CRP level, WBC count, and ANC after therapy did not differ between the PPI and non-PPI groups ( $P=1.000, P=0.668$, and $P=0.668$, respectively). Deterioration in subjective symptoms was observed in the PPI group following therapy; however, there was no significant difference in the occurrence of aggravated symptoms such as 
Table 1. Clinical characteristics of patients with functional abdominal pain in whom stool calprotectin levels were checked

\begin{tabular}{|c|c|c|c|}
\hline Variable & PPI group ( $n=37)$ & Non-PPI group $(n=14)$ & $P$ value \\
\hline Sex, male:female & $11: 26$ & $4: 10$ & 1.000 \\
\hline Age (yr) & $14(5-18)$ & $13(3-17)$ & 0.094 \\
\hline \multicolumn{4}{|c|}{ Laboratory findings before treatment } \\
\hline C-reactive protein (mg/L) & $0.90(0.1-16.9)$ & $1.25(0.8-41.2)$ & 0.079 \\
\hline White blood cell $\left(/ \mathrm{mm}^{3}\right)$ & $7,315(3,650-13,970)$ & $8,490(5,630-16,080)$ & 0.124 \\
\hline Absolute neutrophil count & $4,053(1,707-10,086)$ & $5,782(1,999-11,352)$ & 0.060 \\
\hline \multicolumn{4}{|c|}{ Laboratory findings after treatment } \\
\hline C-reactive protein (mg/L) & $1.10(0.7-37.5)$ & $2.45(0.7-31.8)$ & 0.562 \\
\hline White blood cell $\left(/ \mathrm{mm}^{3}\right)$ & $7,470(3,650-10,060)$ & $10,430(5,950-11,650)$ & 0.133 \\
\hline Absolute neutrophil count & $3,838(1,978-6,161)$ & $4,274(3,294-8,120)$ & 0.460 \\
\hline
\end{tabular}

Values are presented as number or median (range).

PPI, proton pump inhibitor.

Table 2. Comparison of the number of patients with worsened laboratory findings and clinical manifestations before versus after PPI therapy

\begin{tabular}{llll}
\hline Variable & $\begin{array}{c}\text { PPI group } \\
(\mathrm{n}=37)\end{array}$ & $\begin{array}{c}\text { Non-PPl group } \\
(\mathrm{n}=14)\end{array}$ & $P$ value \\
\hline Laboratory findings & & & \\
C-reactive protein & $5(13.5)$ & $2(14.3)$ & 1.000 \\
White blood cell & $5(13.5)$ & $3(21.4)$ & 0.668 \\
Absolute neutrophil count & $5(13.5)$ & $3(21.4)$ & 0.668 \\
Clinical manifestations & & & \\
Abdominal pain & $4(10.8)$ & $0(0)$ & 0.565 \\
Nocturnal abdominal pain & $1(2.7)$ & $0(0)$ & 1.000 \\
Abdominal distension & $0(0)$ & $0(0)$ & - \\
Nausea & $3(8.1)$ & $0(0)$ & 0.552 \\
Vomiting & $1(2.7)$ & $0(0)$ & 1.000 \\
Loose stool & $2(5.4)$ & $0(0)$ & 1.000 \\
Nocturnal defecation & $2(5.4)$ & $1(7.1)$ & 1.000 \\
Bloody stool & $0(0)$ & $0(0)$ & - \\
Tenderness & $1(2.7)$ & $0(0)$ & 1.000 \\
\hline
\end{tabular}

Values are presented as number (\%).

PPI, proton pump inhibitor.

abdominal distension, diarrhea, or abdominal pain between the 2 groups.

\section{Comparison of FC level before and after PPI therapy}

The median FC levels before and after therapy were not significantly different between the 2 groups ( $P=0.527$ and $P=0.113$ ). After therapy, the FC levels decreased to $8.1 \mathrm{mg} / \mathrm{kg}$ (-575.4 to $340.3 \mathrm{mg} /$ $\mathrm{kg}$ ) in the PPI group and increased to $5.6 \mathrm{mg} / \mathrm{kg}(-460.0$ to 186.9 $\mathrm{mg} / \mathrm{kg})$ in the non-PPI group from pretherapy baseline ( $P=0.841)$. There were no differences in the number of patients with increased FC levels between the 2 groups (48.6\% vs. $64.3 \%, P=0.363$ ). The number of patients with an FC level increase of $>50 \mathrm{mg} / \mathrm{kg}$ was not significantly different between the PPI and non-PPI groups (24.3\% and 7.1\%, $P=0.250$ ) (Table 3).

\section{Comparison of FC level according to the duration and type of PPI therapy}

Table 4 shows the comparison of FC levels based on the duration of PPI therapy and type of PPI used.

Of the 37 patients in the PPI group, 25 were treated with the drugs for 4 weeks and 12 were treated for 8 weeks. There were no significant differences in the changes in FC, in patients with increased levels as well as those with levels $>50 \mathrm{mg} / \mathrm{kg}$, based on the duration of PPI therapy ( $P=0.811, P=0.449$, and $P=0.241$, respectively).

In the PPI group, 22 patients were treated with lansoprazole and 15 patients were treated with esomeprazole. There were no significant differences in the changes in FC, in patients with increased levels as well as those with levels $>50 \mathrm{mg} / \mathrm{kg}$, based on the type of PPI used ( $P=0.502, P=0.809$, and $P=0.397$, respectively).

\section{Discussion}

In the present study, the clinical symptoms, laboratory findings, and FC levels of patients who underwent treatment with PPI were not statistically different from those who did not receive PPI. There were no significant differences in FC levels before and after therapy based on the duration and type of PPI used. These results suggest that clinical intestinal inflammation does not occur in patients because of PPI treatment.

Calprotectin is a $36.5-\mathrm{kDa}$ calcium-binding heterodimer consisting of S100A8 (MRP-8) and S100A9 (MRP-14) subunit proteins. ${ }^{15-17)}$ It is mainly derived from neutrophilic granulocytes in the bowel mucosa, and the accumulation of neutrophils results in release of calprotectin in the stool. Calprotectin remains stable in stools for 3-7 days, and since the levels have been shown to correlate with inflammatory activity, analysis of FC has been proposed as a useful marker of bowel inflammation. Several studies have demonstrated the role of FC in the diagnosis and monitoring of the disease activity of IBD. FC has also been suggested as a reliable 
Table 3. Comparison of calprotectin changes with versus without PPI therapy

\begin{tabular}{|c|c|c|c|}
\hline Variable & PPI group $(n=37)$ & Non-PPI group $(n=14)$ & $P$ value \\
\hline Calprotectin before PPI therapy (mg/kg) & $63.6(5.0-626.3)$ & $35.1(10.1-468.1)$ & 0.527 \\
\hline Calprotectin after PPI therapy (mg/kg) & $60.8(0.4-363.3)$ & $29.4(8.1-358.0)$ & 0.113 \\
\hline Changed calprotectin level (mg/kg) & $-8.1(-575.4$ to 340.3$)$ & $5.6(-460.0$ to 186.9$)$ & 0.841 \\
\hline No. of patients with increased calprotectin & $18(48.6)$ & $9(64.3)$ & 0.363 \\
\hline No. of patients with increased calprotectin over 50 mg/kg & $9(24.3)$ & $1(7.1)$ & 0.250 \\
\hline
\end{tabular}

Values are presented as median (range) or number (\%).

PPI, proton pump inhibitor.

Table 4. Comparison of calprotectin changes by PPI therapy duration and type

\begin{tabular}{|c|c|c|c|c|c|c|}
\hline \multirow{2}{*}{ Variable } & \multicolumn{2}{|c|}{ Duration of PPI } & \multirow{2}{*}{$P$ value } & \multicolumn{2}{|c|}{ PPI type } & \multirow{2}{*}{$P$ value } \\
\hline & 4 Weeks $(n=25)$ & 8 Weeks ( $n=12)$ & & Lansoprazole ( $\mathrm{n}=22)$ & Esomeprazole $(n=5)$ & \\
\hline Changed calprotectin level (mg/kg) & $-8.1(-146.7$ to 340.3$)$ & $-3.5(-575.4$ to 199.1$)$ & 0.811 & $4.9(-247.8$ to 199.1$)$ & $-21.4(-575.4$ to 340.3$)$ & 0.502 \\
\hline No. of patients with increased calprotectin & $12(48.0)$ & $6(50.0)$ & 0.449 & $13(59.1)$ & $5(33.3)$ & 0.809 \\
\hline $\begin{array}{l}\text { No. of patients with increased calprotectin over } \\
50 \mathrm{mg} / \mathrm{kg}\end{array}$ & $6(24.0)$ & $3(25.0)$ & 0.241 & $5(22.7)$ & $4(26.7)$ & 0.397 \\
\hline
\end{tabular}

Values are presented as median (range) or number (\%).

PPI, proton pump inhibitor.

tool to screen patients with gut inflammation such as infectious colitis or necrotizing enterocolitis. ${ }^{13-15,17,18)}$ Some publications have shown use for FC in identifying the presence of small bowel inflammation in patients with intestinal inflammation. ${ }^{19-21)}$

Previous studies have suggested that PPI therapy is a risk factor for the development of intestinal problems including bacterial overgrowth. ${ }^{6-9)}$ However, bacterial overgrowth does not cause intestinal inflammation, and the overgrowth alone would not be a significant factor, unless clinically relevant. Therefore, in the present study, we investigated whether PPI therapy caused significant inflammation in the intestine. The results demonstrated that although there were patients who had worsened clinical symptoms, such as abdominal pain, tenderness, and nocturnal defecation after PPI therapy, there were no significant differences between the PPI and non-PPI groups. In addition, there was no significant difference in terms of the evidence of inflammation as assessed by FC levels. From these results, we considered it unlikely that the use of PPI further exacerbated the inflammatory response in the intestines. Additionally, other hematologic tests to assess inflammatory responses such as CRP level, WBC count, and ANC showed no significant differences between the 2 groups.

In addition to intestinal inflammation, there are other side effects of PPI; indeed, there are reports that respiratory infections or otitis media may be increased. ${ }^{6,22,23)}$ There is also an increased risk of polyps or hyperplasia in the stomach following PPI therapy. ${ }^{23)}$ However, the GERD guidelines published by the North American Society for Pediatric Gastroenterology, Hepatology, and the European Society for Pediatric Gastroenterology, Hepatology, and Nutrition suggest that the reported side effects of PPI use, such as upper and lower respiratory infections, constipation, and eczema, are not more common than placebo groups. ${ }^{22)}$ Our results also support the above-mentioned observations. In the GERD guidelines, PPI treatment is usually recommended to last 4 to 8 weeks. In the present study, there was no difference in FC levels based on the duration of treatment.

There are several limitations in our study. First is the lack of randomization to treatment; however, this would not alter the results as the demographic characteristics and baseline laboratory findings, including FC levels, were not different between the PPI and nonPPI groups. Given that there was no significant difference between the 2 groups before and after treatment, this is evidence that there was no difference in the actual levels of FC due to gastric acid suppression treatment. This could be attributed to the fact that PPI treatment was initiated due to more severe symptoms in patients in the PPI group. Therefore, the impact of such a limitation would be minimal. Second, the number of patients included was small and it would have been better if the results of FC were compared with those of previous studies, such as HBT or gastrointestinal inflammatory marker. However, due to the nature of the study conducted in children, it was difficult to involve a greater number of patients and the compliance was inevitably low. Invasive tests were also difficult to perform and could not be compared with well-known tests such as HBT or duodenal aspiration. It would be interesting to conduct further studies comparing the HBT with more participants.

Despite these limitations, in the present study, we attempted to confirm the influence of PPI on intestinal inflammation in pediatric patients as confirmed by clinical manifestations and objectively assessed by measurement of FC levels; however, no significant changes were observed. In the future, further studies will be needed with larger sample sizes and other markers of intestinal inflamma- 
tion as well as analysis of FC.

\section{Conflicts of interest}

No potential conflict of interest relevant to this article was reported.

\section{Acknowledgments}

This study was supported by a 2015 research grant from the Korean Pediatric Society (Seokcheon Research Award).

\section{References}

1. Kim SE, Park HK, Kim N, Joo YE, Baik GH, Shin JE, et al. Prevalence and risk factors of functional dyspepsia: a nationwide multicenter prospective study in Korea. J Clin Gastroenterol 2014;48:e12-8.

2. Ronkainen J, Agréus L. Epidemiology of reflux symptoms and GORD. Best Pract Res Clin Gastroenterol 2013;27:325-37.

3. Forbes D, Lim A, Ravikumara M. Gastroesophageal reflux in the 21st century. Curr Opin Pediatr 2013;25:597-603.

4. Cohen S, Bueno de Mesquita M, Mimouni FB. Adverse effects reported in the use of gastroesophageal reflux disease treatments in children: a 10 years literature review. Br J Clin Pharmacol 2015;80:200-8.

5. Vela MF. Medical treatments of GERD: the old and new. Gastroenterol Clin North Am 2014;43:121-33.

6. McCarthy DM. Adverse effects of proton pump inhibitor drugs: clues and conclusions. Curr Opin Gastroenterol 2010;26:624-31.

7. Fujimori S. What are the effects of proton pump inhibitors on the small intestine? World J Gastroenterol 2015;21:6817-9.

8. Corleto VD, Festa S, Di Giulio E, Annibale B. Proton pump inhibitor therapy and potential long-term harm. Curr Opin Endocrinol Diabetes Obes 2014;21:3-8.

9. Compare D, Pica L, Rocco A, De Giorgi F, Cuomo R, Sarnelli G, et al. Effects of long-term PPI treatment on producing bowel symptoms and SIBO. Eur J Clin Invest 2011;41:380-6.

10. Malfertheiner P, Fass R, Quigley EM, Modlin IM, Malagelada JR, Moss $\mathrm{SF}$, et al. Review article: from gastrin to gastro-oesophageal reflux disease--a century of acid suppression. Aliment Pharmacol Ther
2006;23:683-90.

11. Franco DL, Disbrow MB, Kahn A, Koepke LM, Harris LA, Harrison ME, et al. Duodenal aspirates for small intestine bacterial overgrowth: yield, PPIs, and outcomes after treatment at a tertiary academic medical center. Gastroenterol Res Pract 2015;2015:971582.

12. Sieczkowska A, Landowski P, Zagozdzon P, Kaminska B, Lifschitz C. Small bowel bacterial overgrowth associated with persistence of abdominal symptoms in children treated with a proton pump inhibitor. J Pediatr 2015;166:1310-2.e1.

13. Waugh N, Cummins E, Royle P, Kandala NB, Shyangdan D, Arasaradnam R, et al. Faecal calprotectin testing for differentiating amongst inflammatory and non-inflammatory bowel diseases: systematic review and economic evaluation. Health Technol Assess 2013;17:xvxix, 1-211.

14. Berni Canani R, Rapacciuolo L, Romano MT, Tanturri de Horatio L, Terrin G, Manguso F, et al. Diagnostic value of faecal calprotectin in paediatric gastroenterology clinical practice. Dig Liver Dis 2004;36: 467-70.

15. Bonnín Tomàs A, Vila Vidal M, Rosell Camps A. Fecal calprotectin as a biomarker to distinguish between organic and functional gastrointestinal disease. Rev Esp Enferm Dig 2007;99:689-93.

16. Ezri J, Nydegger A. Pediatrics. Fecal calprotectin in children: use and interpretation. Rev Med Suisse 2011;7:69-70.

17. Rodrigo L. Fecal calprotectin. Rev Esp Enferm Dig 2007;99:683-8.

18. Jang HJ, Park JH, Kim CS, Lee SL, Lee WM. Amino acid-based formula in premature infants with feeding intolerance: comparison of fecal calprotectin level. Pediatr Gastroenterol Hepatol Nutr 2018;21: 189-95.

19. Höög CM, Bark LÅ, Broström O, Sjöqvist U. Capsule endoscopic findings correlate with fecal calprotectin and C-reactive protein in patients with suspected small-bowel Crohn's disease. Scand J Gastroenterol 2014;49:1084-90.

20. Olsen PA, Fossmark R, Qvigstad G. Fecal calprotectin in patients with suspected small bowel disease--a selection tool for small bowel capsule endoscopy? Scand J Gastroenterol 2015;50:272-7.

21. Montalto M, Santoro L, Dalvai S, Curigliano V, D’Onofrio F, Scarpellini E, et al. Fecal calprotectin concentrations in patients with small intestinal bacterial overgrowth. Dig Dis 2008;26:183-6.

22. Rosen R, Vandenplas Y, Singendonk M, Cabana M, DiLorenzo C, Gottrand F, et al. Pediatric gastroesophageal reflux clinical practice guidelines: Joint Recommendations of the North American Society for Pediatric Gastroenterology, Hepatology, and Nutrition and the European Society for Pediatric Gastroenterology, Hepatology, and Nutrition. J Pediatr Gastroenterol Nutr 2018;66:516-54.

23. Owen DA. Gastritis and carditis. Mod Pathol 2003;16:325-41. 